\title{
Increased Cerebrospinal Fluid Levels of Ubiquitin Carboxyl-Terminal Hydrolase L1 in Patients with Alzheimer's Disease
}

\author{
Annika Öhrfelt ${ }^{a}$ Per Johansson ${ }^{b, d}$ Anders Wallin $^{a}$ Ulf Andreasson ${ }^{a}$ \\ Henrik Zetterberg ${ }^{a}$ e Kaj Blennow ${ }^{a}$ Johan Svensson ${ }^{c, d}$ \\ ${ }^{a}$ Clinical Neurochemistry Laboratory, Department of Psychiatry and Neurochemistry, \\ Institute of Neuroscience and Physiology, Sahlgrenska University Hospital Mölndal, \\ Sahlgrenska Academy, University of Gothenburg, Mölndal, ${ }^{b}$ Department of \\ Neuropsychiatry, Skaraborg Hospital, Falköping, 'Department of Endocrinology, Skaraborg \\ Hospital, Skövde, and dinstitute of Medicine, Sahlgrenska Academy, University of \\ Gothenburg, Gothenburg, Sweden; eUCL Institute of Neurology, London, UK
}

\section{Key Words}

Alzheimer's disease - Biomarkers - Cerebrospinal fluid · DJ-1 - Neuron-specific enolase · Ubiquitin carboxyl-terminal hydrolase L1 · Tau phosphorylated at threonine 231

\begin{abstract}
Background: Dysfunctions of the ubiquitin proteasome system (UPS), including the highly abundant neuronal enzyme ubiquitin carboxyl-terminal hydrolase L1 (UCH-L1), and autophagy-related changes (lysosomal degradation) are implicated in several neurodegenerative disorders including Alzheimer's disease (AD). Method: This study evaluated cerebrospinal fluid (CSF) levels of UCH-L1, protein deglycase (DJ-1), neuron-specific enolase (NSE), and tau phosphorylated at threonine 231 (P-tau $\left.{ }_{231}\right)$ in two independent patient and control cohorts. Cohort 1 included CSF samples from subjects having an AD biomarker profile $(n=10)$ or a control biomarker profile $(n=31)$, while cohort 2 was a monocenter clinical study including patients with $A D(n=32)$, mild cognitive impairment $(n=13)$, other dementias $(n=15)$, as well as cognitively healthy controls $(n=20)$. Results: $U C H-L 1$ and P-tau ${ }_{231}$ were elevated in AD patients compared to controls in both cohorts. CSF levels of DJ-1 and NSE were unchanged in the AD group, whereas they were decreased in the group of other dementia compared to controls in the clinical study. Conclusion: Our main findings support that the UPS pathway may be impaired in AD, and UCH-L1 may serve as an additional CSF biomarker for AD.
\end{abstract}




\section{Background}

The ubiquitin proteasome system (UPS) selectively degrades proteins targeted for degradation by covalent conjugation to ubiquitin [1]. When these proteins have been linked to the ubiquitin chain, they are directed to degradation via the UPS [1] or in the lysosome [2]. Alzheimer's disease (AD) is a protein-misfolding disease characterized by accumulation of amyloid $\beta(\mathrm{A} \beta$ ) peptides and hyperphosphorylated tau protein into plaques and neurofibrillary tangles, respectively [3]. The ubiquitin protein is also accumulated in these structural AD changes [4-7]. This suggests that dysfunction of the quality control mechanisms regulating protein breakdown, including both the UPS and the lysosome, might be directly or indirectly involved in the pathogenesis of AD.

Ubiquitin carboxyl-terminal hydrolase L1 (UCH-L1; also called neuron cytoplasmic protein 9.5 and PGP 9.5) is a highly abundant neuron-specific cytoplasmic enzyme [8-10]. It modifies the activity of the UPS by acting as a deubiquitinating hydrolase [10], ubiquitin ligase [11], and a monoubiquitin stabilizer [12]. UCH-L1 [13] and ubiquitin [4-7] are found in $\mathrm{A} \beta$ plaques and neurofibrillary tangles in the AD brain, supporting evidence for UPS dysfunction in AD. Furthermore, in an animal model, dysfunction of UCH-L1 affected the biological function of the tau protein as well as phosphorylation of tau [14]. Genetic studies demonstrate a link between the UCH-L1 (PARK5) gene and rare familiar forms of Parkinson's disease (PD) [15], and most previous studies have shown a protective effect of the S18Y polymorphism (rs id 5030732) against sporadic PD [16, 17]. There are only a few reports regarding the implications of genetic variation in UCH-L1 in AD $[18,19]$, and there are conflicting results in terms of the role of the UCH-L1 polymorphism in AD patients [20-22]. UCH-L1 levels in the cerebrospinal fluid (CSF) of AD patients have, to our knowledge, not been reported, whereas several studies have found an increased CSF ubiquitin level in AD [23-26].

In the CSF, the fluid that surrounds the central nervous system, the AD core biomarkers total-tau (T-tau), tau phosphorylated at threonine $181\left(\mathrm{P}-\operatorname{tau}_{181}\right)$, and $\mathrm{A} \beta_{1-42}$ are thought to reflect neurodegeneration, neurofibrillary tangles, and aggregation of $A \beta$ into plaques, respectively [27]. Most studies confirm a typical AD biomarker signature in AD with elevated T-tau and P-tau in addition to reduced levels of $A \beta_{1-42}$. Recently, the AD core CSF biomarkers have been included in the research criteria for the diagnosis of both early and manifest AD by the International Working Group [28] and in the diagnostic guidelines from the National Institute on Aging-Alzheimer's Association [29], respectively. However, the diagnostic performance of CSF tau phosphorylated at threonine 231 (P-tau 231 ) compared to that of CSF P-tau 181 is not well known, although in a recent study $\mathrm{P}-\mathrm{tau}_{231}$ displayed a greater overall specificity for $\mathrm{AD}$ than P-tau 181 [30].

The protein deglycase (DJ-1) (PARK7) gene is linked to PD [31]. Although the role of DJ-1 has not fully been evaluated, it could provide protection from oxidative stress [32]. In previous studies, CSF DJ-1 levels were unchanged in AD [33, 34]. Neuron-specific enolase (NSE) is a glycolytic enzyme present in neuronal and neuroendocrine cells and might be a marker of damage to cortical nonmyelinated neurons [27, 35]. Several previous studies have shown conflicting results with reduced [36], increased [35, 37, 38], or unchanged [39, 40] CSF levels in $\mathrm{AD}$ patients compared to controls.

In this study, a commercially available magnetic bead panel for neurological disorders was initially evaluated using CSF samples from subjects having an AD core biomarker profile or a control core biomarker profile, respectively. The neurological panel was then used to assess the CSF levels of UCH-L1, P-tau $231, \mathrm{DJ}-1$, and NSE in a well-characterized monocenter cohort of patients with cognitive impairment and matched healthy controls [41]. 
Table 1. Demographic data and biomarker levels from the pilot study for the patients with AD and controls based on the biomarker profile
Öhrfelt et al.: Increased Cerebrospinal Fluid Levels of Ubiquitin Carboxyl-Termina Hydrolase L1 in Patients with Alzheimer's Disease

\begin{tabular}{|c|c|c|}
\hline & $\begin{array}{l}\text { Control biomarker } \\
\text { profile }\end{array}$ & $\begin{array}{l}\text { AD biomarker } \\
\text { profile }\end{array}$ \\
\hline Subjects (men/women), $\mathrm{n}$ & $31(16 / 15)$ & $10(3 / 7)$ \\
\hline Age, years & $72(70-79)$ & $79(73-85)$ \\
\hline $\mathrm{A} \beta_{1-42}, \mathrm{ng} / \mathrm{l}$ & $779(636-991)$ & $354(320-414)^{b}$ \\
\hline T-tau, ng/l & $276(170-332)$ & $1,040(665-1,110)^{\mathrm{b}}$ \\
\hline P-tau $181, n g / l$ & $43(28-49)$ & $101(85-147)^{b}$ \\
\hline UCH-L1, $\mu \mathrm{g} / \mathrm{l}$ & $4.5(3.8-5.3)$ & $12(7.6-14)^{b}$ \\
\hline P-tau $231, \mathrm{pM}$ & $406(314-495)$ & $3,810(2,870-5,070)^{b}$ \\
\hline $\mathrm{DJ}-1, \mu \mathrm{g} / \mathrm{l}$ & $17(9.3-31)$ & $37(26-52)^{a}$ \\
\hline NSE, $\mu \mathrm{g} / \mathrm{l}$ & $23(16-29)$ & $42(38-56)^{b}$ \\
\hline
\end{tabular}

Data are given as median (interquartile range) unless otherwise indicated. Statistical differences were determined using nonparametric tests. ${ }^{\mathrm{a}} \mathrm{p}<0.01,{ }^{\mathrm{b}} \mathrm{p}<0.0001$ vs. control.

\section{Materials and Methods}

\section{CSF Samples of the Pilot Study}

An initial pilot study was performed using decoded human CSF samples supplied by the Clinical Neurochemistry Laboratory, Sahlgrenska University Hospital, Mölndal. Patients were designated as control or $\mathrm{AD}$ according to CSF AD core biomarker levels using in-house optimized cutoff levels of $>90 \%$ specific for AD [42]: T-tau $>400 \mathrm{ng} / \mathrm{l}, \mathrm{P}$-tau $181>80 \mathrm{ng} / \mathrm{l}$, and $\mathrm{A} \beta_{1-42}<550 \mathrm{ng} / \mathrm{l}$. The age-matched test material included 10 patients with an AD biomarker profile and 31 subjects with a control biomarker profile (table 1 ).

\section{CSF Samples of the Clinical Study}

All CSF samples in the clinical study were collected by lumbar puncture in the L3/L4 or L4/L5 interspace at the standardized time point 8:30 to 9:00 a.m. The first $12 \mathrm{ml}$ of CSF was collected in a polypropylene tube and immediately transported to the local laboratory for centrifugation at $2,000 \mathrm{~g}$ at $+4^{\circ} \mathrm{C}$ for $10 \mathrm{~min}$. The supernatant was pipetted off, gently mixed to avoid possible gradient effects, and aliquoted in polypropylene tubes that were stored at $-80^{\circ} \mathrm{C}$ pending biochemical analyses, without being thawed and refrozen.

The study participants as well as the AD CSF biomarker data in the clinical study have been reported previously [41,43-46]. The study consisted of 60 patients (30 men and 30 women, all of Caucasian origin) admitted by their general practitioner for evaluation of cognitive impairment to a memory clinic in the region of Västra Götaland, Sweden. The patients were examined by a single specialized physician (P.J.) in 2000-2008. Inclusion criteria, besides being referred for evaluation of suspected dementia, were age 65-80 years, a body mass index (BMI) of 20-26, and a waist:hip ratio of 0.65-0.90 in women and 0.70-0.95 in men. Exclusion criteria were serum creatinine $>175 \mathrm{~mm}$, diabetes mellitus, previous myocardial infarction, malignancy including brain tumor, subdural hematoma, ongoing alcohol abuse, medication with cortisone, and previous or present medication with acetylcholine esterase inhibitors. The study also included age-matched healthy controls (10 men and 10 women) recruited contemporaneously from the same geographical area among spouses of the included patients and by advertisements in local newspapers. The control subjects had no subjective symptoms of cognitive dysfunction and had similar exclusion criteria as the patients. 
All diagnoses were assessed by an independent specialized physician, as previously described [41]. The presence or absence of dementia was diagnosed according to the Diagnostic and Statistical Manual of Mental Disorders, Fourth Edition (DSM-IV), criteria. Patients with dementia were classified as suffering from $\mathrm{AD}$ [47] or vascular dementia (VaD) according to the requirements by NINDS-AIREN [48] or the guidelines by Erkinjuntti et al. [49] for the subcortical type of VaD. Frontotemporal dementia, PD dementia, and dementia with Lewy bodies (DLB) were diagnosed as described previously [41].

Mild cognitive impairment (MCI) was diagnosed in the clinical setting in patients with cognitive impairment who did not fulfill the criteria for dementia [50]. Patients with MCI were followed at least annually for a median of 3 (range 1-7) years to evaluate whether they later developed dementia. During the follow-up visits, $13 \mathrm{MCI}$ patients remained in stable cognitive function (sMCI). Others progressed to dementia during the follow-up period and were diagnosed with $\operatorname{AD}(n=7), \operatorname{VaD}(n=3)$, or frontotemporal lobe dementia $(n=1)$. MCI patients diagnosed with $A D$ on follow-up visits did not differ in CSF levels of $A \beta_{1-42}$, T-tau, or P-tau from patients with established AD at baseline. In total, the study population consisted of patients with AD dementia or with MCI diagnosed with AD dementia upon follow-up ( $\mathrm{n}=$ $32)$, patients with sMCI $(n=13)$, patients with other dementias $(n=15)$, and healthy controls $(\mathrm{n}=20)$. The distribution of diagnoses in the other dementia group were VaD or MCI diagnosed with VaD upon follow-up ( $\mathrm{n}=10)$, DLB $(\mathrm{n}=4)$, and MCI that later converted to frontotemporal lobe dementia $(n=1)$. Before the test day, a mini-mental state examination (MMSE) [51] was performed.

\section{CSF Analyses}

Measurements of the core AD biomarkers $\left(A \beta_{1-42}, \mathrm{~T}\right.$-tau, and P-tau $\left.{ }_{181}\right)$ were performed using commercially available assays from Fujirebio, Ghent, Belgium [INNOTEST ${ }^{\circledR}$ $\beta$-AMYLOID(1-42), INNOTEST ${ }^{\circledR}$ hTAU Ag, and INNOTEST ${ }^{\circledR}$ PHOSPHO-TAU(181P)]. For the clinical study, the core AD biomarkers were analyzed on one occasion using the same batch of reagents, which has previously been reported [41, 43-46]. Furthermore, for the clinical study, CSF hemoglobin concentrations were measured using a human hemoglobin ELISA kit (Bethyl Laboratories, Inc.) according to the manufacturer's protocol. For the clinical study, red blood cells (RBCs) were counted in most of the samples. Hemoglobin levels above 1,000 ng/l [52] and/or more than 500 erythrocytes per $\mu$ l were indicative of significant blood contamination. Only two of the $80 \mathrm{CSF}$ samples in the clinical study fulfilled these criteria. However, these two samples were not excluded from the study since statistical analyses showed that our results were not affected by these two samples (data not shown).

MILLIPLEX MAP Human Neurological Disorders Magnetic Bead Panel 1 HND1MAG-39K (Merck Millipore) was used for quantification of UCH-L1 (PARK5), DJ-1 (PARK7), NSE, P-tau 231 , NGF- $\beta$, and $\alpha$-synuclein in accordance with the protocol provided by the manufacturer, and $25 \mu \mathrm{l}$ neat CSF was analyzed. DJ-1 levels $<4.8 \mu \mathrm{g} / \mathrm{l}$ and levels of NSE $>60 \mu \mathrm{g} / \mathrm{l}$ were set to 4.8 and $60 \mu \mathrm{g} / \mathrm{l}$, respectively. Samples were analyzed on a MAGPIX ${ }^{\circledR}$ system (Merck Millipore). Quality control (QC) samples (QC1 and QC2) analyzed in duplicate supplied by the manufacturer fulfilled the specified concentration levels. Coefficients of variation were $<8 \%$ for all analytes. The levels of NGF- $\beta$ and $\alpha$-synuclein are not reported since they were below the detection limit in all CSF samples of both the pilot study and the clinical study.

\section{Statistical Analysis}

Because the distribution of most analytes was skewed (Shapiro-Wilk test, $\mathrm{p}<0.05$ ), nonparametric statistics were used for the statistical analysis using SPSS version 20.0 statistical software (SPSS Inc., Chicago, Ill., USA). Data are given, if not otherwise stated, as the median (interquartile range). Differences between more than two groups were assessed with 
Öhrfelt et al.: Increased Cerebrospinal Fluid Levels of Ubiquitin Carboxyl-Terminal Hydrolase L1 in Patients with Alzheimer's Disease

Table 2. Demographic data and biomarker levels for the clinical study

\begin{tabular}{lcccc}
\hline & Controls & sMCI & AD & Other dementia \\
\hline Subjects (men/women), $\mathrm{n}$ & $20(10 / 10)$ & $13(5 / 8)$ & $32(15 / 17)$ & $15(10 / 5)$ \\
Age, years & $75(70-78)$ & $72(69-74)$ & $75(71-77)$ & $74(72-77)$ \\
MMSE & $29(27-29)^{\mathrm{f}}$ & $29(27-29)^{\mathrm{f}}$ & $23(19-25)^{\mathrm{c}}$ & $24(20-26)^{\mathrm{c}}$ \\
$\mathrm{A} \beta_{1-42}, \mathrm{ng} / \mathrm{l}$ & $992(786-1,038)^{\mathrm{f}}$ & $671(544-833)^{\mathrm{a}, \mathrm{f}}$ & $420(336-493)^{\mathrm{c}}$ & $404(354-816)^{\mathrm{a}}$ \\
T-tau, ng/l & $327(223-398)^{\mathrm{f}}$ & $270(230-390)^{\mathrm{f}}$ & $584(434-747)^{\mathrm{c}}$ & $311(260-380)^{\mathrm{f}}$ \\
P-tau ${ }_{181}, \mathrm{ng} / \mathrm{l}$ & $65(50-79)^{\mathrm{f}}$ & $60(38-74)^{\mathrm{f}}$ & $98(78-113)^{\mathrm{c}}$ & $47(36-63)^{\mathrm{a}, \mathrm{f}}$ \\
UCH-L1, $\mu \mathrm{g} / \mathrm{l}_{\text {P-tau }}^{231, \mathrm{pM}}$ & $7.2(6.2-8.9)^{\mathrm{f}}$ & $7.4(5.1-8.9)^{\mathrm{e}}$ & $11(8.7-13)^{\mathrm{c}}$ & $7.2(5.8-9)^{\mathrm{f}}$ \\
DJ-1, $\mu \mathrm{g} / \mathrm{l}$ & $1,000(900-1,200)^{\mathrm{f}}$ & $1,200(700-1,700)^{\mathrm{f}}$ & $3,400(2,700-3,850)^{\mathrm{c}}$ & $1,500(1,100-1,800)^{\mathrm{f}}$ \\
NSE, $\mu \mathrm{g} / \mathrm{l}$ & $19(16-20)$ & $14(12-16)$ & $17(13-19)$ & $7.1(6.3-18)^{\mathrm{b}, \mathrm{d}}$ \\
\hline
\end{tabular}

Data are given as median (interquartile range) unless otherwise indicated. Statistical differences were determined using nonparametric tests. The demographic data and the core AD biomarkers have previously been reported $[41,43-46]$. ${ }^{\mathrm{a}} \mathrm{p} \leq 0.05$, ${ }^{b} p \leq 0.001,{ }^{c} p \leq 0.0001$ vs. control; ${ }^{d} p \leq 0.05,{ }^{e} p \leq 0.001,{ }^{f} p \leq 0.0001$ vs. AD.

the Kruskal-Wallis test. If statistically significant $(\mathrm{p}<0.05)$, the Mann-Whitney U test was then used for pairwise comparisons. The diagnostic value of each biomarker was assessed using receiver operating characteristic (ROC) curves with values of the area under the curve (AUC) and a 95\% confidence interval (CI) calculated using GraphPad Prism 5 (GraphPad Software Inc., La Jolla, Calif., USA). The correlation coefficients ( $\rho$ ) were calculated using the Spearman two-tailed correlation test. Significance was obtained if the two-tailed $\mathrm{p}$ value was $<0.05$.

\section{Statement of Ethics}

The study was approved by the ethical committee of the University of Gothenburg, and informed consent was obtained from all participants. The study was conducted according to the Declaration of Helsinki.

\section{Results}

\section{Pilot Study}

In the pilot study, CSF levels of all the analytes (UCH-L1, DJ-1, NSE, and P-tau 231 ) were significantly higher in the group with an AD biomarker profile $(n=10)$ than in the group with a control biomarker profile $(\mathrm{n}=31)$ (table 1$)$.

\section{Clinical Samples}

Demographic Results and CSF AD Biomarkers

Table 2 shows the demographic characteristics of the groups. In the clinical cohort, patients and controls were comparable in terms of age, gender, BMI, and waist:hip ratio. Patients with AD and other dementia had both significantly lower MMSE scores compared to controls. The core AD biomarkers for the clinical study have previously been reported [41, 43-45]. The AD group showed significantly higher CSF levels of T-tau and P-tau 181 than the control group, while the $A \beta_{1-42}$ concentration was significantly decreased (table 2). CSF levels of P-tau 181 and $A \beta_{1-42}$ were also significantly altered in the other dementia group (table 2). 


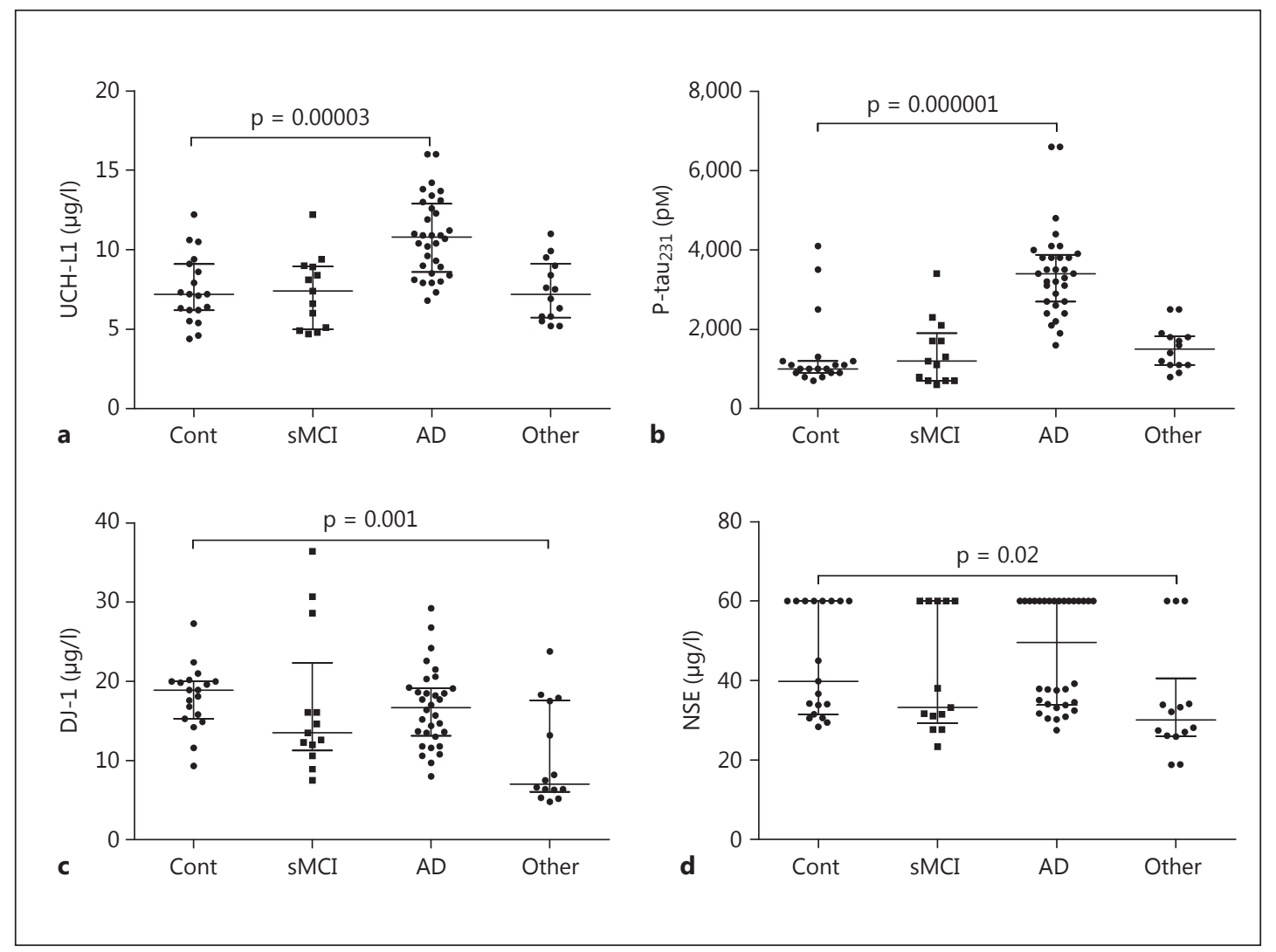

Fig. 1. Individual values for UCH-L1 (a), P-tau 231 (b), DJ-1 (c), and NSE (d) in CSF samples from healthy controls (cont) and patients with sMCI, AD, and other dementias (other). The lower, upper, and middle lines of the error bars correspond to the 25 th and 75 th percentiles and the medians, respectively.

\section{CSF Concentrations of UCH-L1, P-Tau 231, DJ-1, and NSE}

The CSF levels of UCH-L1 and P-tau 231 were significantly increased in patients with AD compared to controls, while these biomarkers were unaltered in other dementia and SMCI (fig. 1a, b; table 2). The CSF levels of DJ-1 and NSE were unchanged in the AD group, whereas they were significantly decreased in the group of other dementia compared to controls (fig. 1c, d; table 2).

\section{ROC Curve Analysis}

UCH-L1 and P-tau 231 could differentiate AD from controls with an AUC of 0.854 (95\% CI 0.746-0.963; $\mathrm{p}<0.0001$ ) and 0.915 (95\% CI 0.813-1.018; $\mathrm{p}<0.0001$ ), respectively (fig. 2). The AUC for the core AD biomarkers $A \beta_{1-42}$, T-tau, and P-tau 181 were 0.938 (95\% CI 0.8651.010 ; $<$ < 0.0001), 0.909 (95\% CI 0.833-0.986; p < 0.0001), and 0.844 (95\% CI 0.736-0.954; $\mathrm{p}<0.0001$ ), respectively (fig. 2). DJ-1 and NSE could differentiate other dementia from controls with an AUC of 0.835 (95\% CI 0.679-0.990; $\mathrm{p}=0.001$ ) and 0.744 (95\% CI 0.562 $0.927 ; p=0.02$ ), respectively.

Correlation Analysis

None of the investigated CSF biomarkers correlated with age, MMSE score, or hemoglobin concentration in either the control group or in patients with AD (table 3). The levels of UCH-L1 correlated positively with the levels of P-tau 181, P-tau 231, DJ-1, and NSE in both the 
Fig. 2. ROC curve analysis for UCH-L1 (black), P-tau 231 (purple), $\mathrm{A} \beta_{1-42}$ (blue), T-tau (yellow), and $\mathrm{P}$-tau 181 (orange) in CSF samples for differentiation of AD patients $(\mathrm{n}=32)$ from controls $(\mathrm{n}=20)$ in the clinical study. AUC were 0.854 (95\% CI 0.746-0.963; $\mathrm{p}<0.0001)$, 0.915 (95\% CI 0.813-1.018; $\mathrm{p}<$ $0.0001), 0.938$ (95\% CI $0.865-$ $1.010 ; \mathrm{p}<0.0001), 0.909(95 \% \mathrm{CI}$ $0.833-0.986 ; \mathrm{p}<0.0001$ ), and 0.844 (95\% CI 0.736-0.954; $\mathrm{p}<$ $0.0001)$, respectively.

Öhrfelt et al.: Increased Cerebrospinal Fluid Levels of Ubiquitin Carboxyl-Terminal Hydrolase L1 in Patients with Alzheimer's Disease

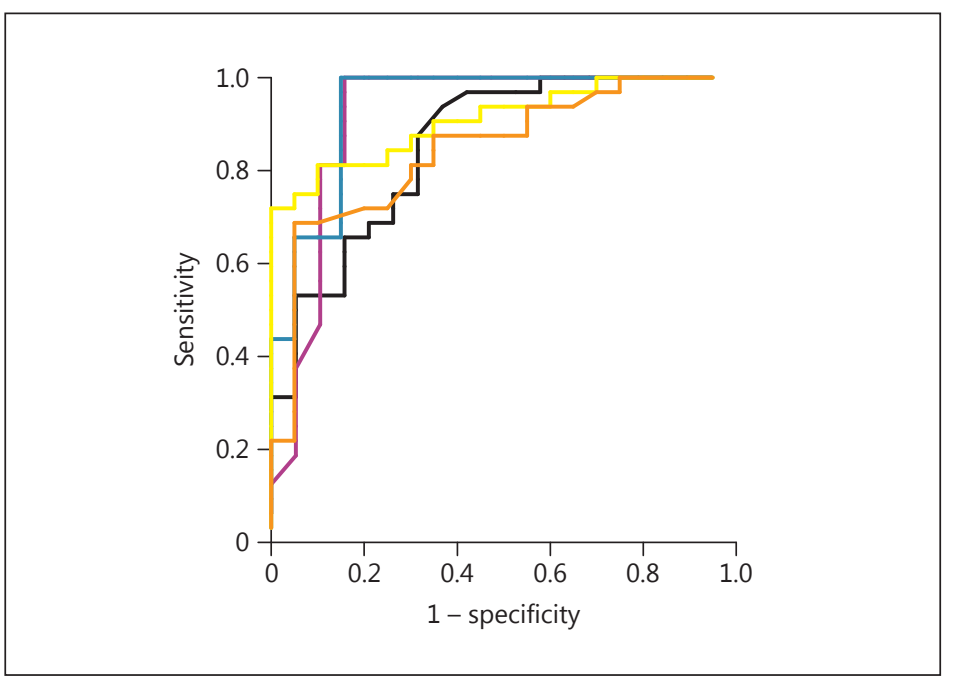

Table 3. Correlation between age, MMSE, hemoglobin, and biomarker levels for the clinical study

\begin{tabular}{|c|c|c|c|c|}
\hline & UCH-L1 & P-tau 231 & DJ-1 & NSE \\
\hline \multicolumn{5}{|l|}{ Controls $(n=20)$} \\
\hline Age & n.s. & n.s. & n.s. & n.s. \\
\hline MMSE & n.s. & n.s. & n.s. & n.s. \\
\hline Hemoglobin & n.s. & n.s. & n.s. & n.s. \\
\hline $\mathrm{A} \beta_{1-42}$ & n.s. & n.s. & n.s. & n.s. \\
\hline T-tau & n.s. & $0.792^{c}$ & n.s. & n.s. \\
\hline P-tau 181 & $0.698^{\mathrm{c}}$ & $0.835^{c}$ & $0.580^{\mathrm{b}}$ & \\
\hline UCH-L1 & & $0.588^{\mathrm{b}}$ & $0.506^{\mathrm{a}}$ & $0.617^{\mathrm{b}}$ \\
\hline P-tau 231 & & & n.s. & n.s. \\
\hline DJ-1 & & & & n.s. \\
\hline \multicolumn{5}{|l|}{$\mathrm{AD}(\mathrm{n}=32)$} \\
\hline Age & n.s. & n.s. & n.s. & n.s. \\
\hline MMSE & n.s. & n.s. & n.s. & n.s. \\
\hline Hemoglobin & n.s. & n.s. & n.s. & n.s. \\
\hline $\mathrm{A} \beta_{1-42}$ & n.s. & n.s. & $0.383^{\mathrm{a}}$ & n.s. \\
\hline T-tau & $0.588^{\mathrm{c}}$ & $0.894^{c}$ & $0.673^{c}$ & $0.652^{\mathrm{c}}$ \\
\hline P-tau ${ }_{181}$ & $0.526^{\mathrm{b}}$ & $0.921^{\mathrm{c}}$ & $0.636^{c}$ & $0.618^{c}$ \\
\hline UCH-L1 & & $0.491^{\mathrm{b}}$ & $0.658^{c}$ & $0.522^{\mathrm{b}}$ \\
\hline P-tau 231 & & & $0.597^{c}$ & $0.512^{\mathrm{b}}$ \\
\hline DJ-1 & & & & $0.400^{\mathrm{a}}$ \\
\hline $\begin{array}{r}\text { Correlation } \\
\text { coefficient }(\rho) \\
\text { reported. }{ }^{a} p \leq\end{array}$ & $\begin{array}{l}\text { by } \\
\text { ant }(1 \\
01,{ }^{c} p\end{array}$ & $\begin{array}{l}\text { Spearm } \\
>0.05 \\
1 .\end{array}$ & rank & tion \\
\hline
\end{tabular}

control group and in AD patients (table 3). Moreover, UCH-L1 levels correlated positively with T-tau in the AD group but not in the control group (table 3). The levels of P-tau 231 correlated positively with the CSF levels DJ-1 and NSE in AD patients but not in controls (table 3). Finally, the CSF levels of P-tau 231 correlated positively with P-tau 181 and T-tau both in the control group and in patients with $\mathrm{AD}$ (table 3 ). 


\section{Discussion}

In both investigated CSF materials, we found that the levels of UCH-L1 and P-tau 231 were significantly increased in AD patients compared to controls, while these biomarkers were unaltered in other dementias and SMCI. Moreover, the separation of AD from controls was of similar magnitude when using these markers as when using the CSF core AD biomarkers $\left(\mathrm{A} \beta_{1-42}, \mathrm{~T}-\mathrm{tau}\right.$, and P-tau $\left.{ }_{181}\right)$. In the clinical study, CSF levels of DJ-1 and NSE were unchanged in the $\mathrm{AD}$ group, whereas they were decreased in the group of other dementias compared to the controls.

To our knowledge, this is the first study to assess the potential of UCH-L1 as a CSF biomarker for AD. UCH-L1, a neuronal-specific enzyme that is highly abundant in the brain [8-10], is one of the enzymes involved in the regulation of proteosomal degradation. During this process, normal proteins with short half-life or misfolded proteins are conjugated with ubiquitin, which destines them for proteosomal [1,10-12] and/or lysosomal degradation [2]. The UPS has previously been implicated in the pathogenesis of AD, and UCH-L1 is present in neurofibrillary tangles in the AD brain $[13,53]$. Several previous studies have reported increased CSF ubiquitin levels in AD patients [23-26], but little has previously been known of the CSF UCH-L1 level in AD. Our results show that the CSF UCH-L1 level is increased in AD and that CSF UCH-L1 can separate AD patients from controls with high diagnostic accuracy in a ROC curve analysis.

The CSF UCH-L1 level did not only correlate with CSF levels of T-tau and P-tau, but there was also a positive correlation between CSF levels of UCH-L1 and NSE both in the AD group and in the controls. Both T-tau and NSE have previously been suggested to be general markers of damage to cortical nonmyelinated neurons $[27,35]$, which might indicate that our finding of an elevated CSF UCH-L1 level in AD to some extent reflects neurodegeneration. Recent reports suggest that UCH-L1 is released after brain damage caused by acute neurological insults such as traumatic brain injury and subarachnoid hemorrhage [54, 55]. Therefore, the diagnostic accuracy of CSF UCH-L1 to separate AD from brain damage of other causes needs to be investigated in further studies. In contrast, P-tau might be a more specific marker for AD [27], since high CSF levels of P-tau have been found to correlate with the accumulation of cortical neurofibrillary tangles $[56,57]$. Thus, the positive correlation between CSF UCH-L1 and CSF P-tau found in our study combined with previous findings that neurofibrillary tangles in $\mathrm{AD}$ are ubiquitinated [4-7], and that the numbers of neurofibrillary tangles in AD brains relate negatively to the level of soluble UCH-L1 [18], support the hypothesis that the elevated UCH-L1 levels in AD could reflect a higher expression of UPS enzymes to compensate for a higher load of misfolded proteins. Furthermore, dysfunction of UCH-L1 in an animal model affected the biological function of tau as well as the phosphorylation of tau [14].

The elevated P-tau 231 level in the AD group is in concordance with previous reports that both P-tau 231 and P-tau 181 are increased in AD [58-60]. We also confirm that CSF levels of P-tau 231 and P-tau 181 correlate tightly [58], suggesting that these P-tau epitopes reflect the same pathogenic process and may be used interchangeably to measure brain neurofibrillary tangle load $[56,57]$. The results of a recent study suggested that $P-t_{2 a u}{ }_{231}$ has a greater overall specificity for $\mathrm{AD}$ compared to P-tau 181 [30]. However, another study found a similar performance for P-tau ${ }_{231}$ and P-tau 181 in differentiating $\mathrm{AD}$ patients from controls, while P-tau ${ }_{181}$ performed better in differentiating AD from Lewy body dementia and P-tau 231 performed better in differentiating AD from frontotemporal dementia [58]. Further studies are warranted to settle whether there is a difference in the diagnostic performance of P-tau $\mathrm{t}_{231}$ and P-tau $\mathrm{t}_{181}$ to identify $\mathrm{AD}$ and other neurodegenerative disorders.

DJ-1 (PARK7) is genetically linked to PD [31]. Even though the physiological function of DJ-1 has not fully been evaluated, it is thought to play a protective role during oxidative stress 
[32]. In accordance with previous reports, we found that the DJ-1 level was not changed in AD compared to controls [33,34]. NSE is a glycolytic enzyme present in neuronal and neuroendocrine cells and is associated with neurodegeneration [27,35]. Previous studies have shown conflicting results with reduced [36], increased [35, 37, 38], or unchanged $[39,40]$ CSF NSE levels in AD compared to controls. In the present study, CSF levels of NSE were unaltered in $\mathrm{AD}$, whereas in patients with other dementias CSF levels of NSE as well as of DJ-1 were reduced. However, the other dementia group was heterogeneous with relatively few cases of each specific diagnosis such as VaD and DLB. Therefore, further studies are needed to explore the roles of NSE and DJ-1 in dementias other than AD.

The present study represents the monocenter design of the clinical study with strictly defined procedures regarding lumbar puncture and laboratory assays. Patients and controls were matched in terms of age, gender, BMI, and waist:hip ratio, and none of the participants had diabetes mellitus or received treatment with acetylcholine esterase inhibitors or glucocorticoids. One limitation of the clinical study is the cross-sectional design, and changes over time could therefore not be studied. Furthermore, the lack of separation between AD patients and controls in terms of CSF NSE levels might be explained by NSE levels exceeding the highest allowed level being set to the highest standard concentration. Moreover, since both DJ-1 and NSE are abundant in RBCs, their CSF levels could be falsely elevated due to blood contamination $[33,61]$. We investigated RBC contamination by assessment of the hemoglobin levels and RBC counting and found that only two samples were confounded by RBC contamination. These two samples were not excluded from the study since additional statistical analyses showed that these samples did not affect the final CSF result of DJ-1 and NSE. In addition, neither DJ-1 nor NSE correlated with CSF hemoglobin. Finally, the evaluated magnetic bead panel was not able to measure NGF- $\beta$ or $\alpha$-synuclein in any of the analyzed CSF samples. The QC samples provided by the manufacturer fulfilled the specified concentration levels, suggesting that the multiassay was not sufficiently sensitive to measure NGF- $\beta$ and $\alpha$-synuclein in our CSF samples.

\section{Conclusions}

In this study, we evaluated a magnetic bead panel for neurological disorders. CSF UCH-L1 and $\mathrm{P}$-tau ${ }_{231}$ levels were elevated in patients with $\mathrm{AD}$ compared to healthy controls. The elevated CSF levels of UCH-L1 might indirectly reflect disturbed proteosomal degradation or that it is released in response to general neurodegeneration in AD. In addition, the clinical study suggests UCH-L1 to be an additional CSF biomarker for AD and that CSF P-tau 231 has a high diagnostic accuracy for $\mathrm{AD}$, suggesting that CSF P-tau ${ }_{231}$ is a valid alternative to CSF P-tau ${ }_{181}$. CSF levels of DJ-1 and NSE were decreased in the other dementia group, but this group was relatively small, and further studies are needed to clarify the role of DJ-1 and NSE in dementing disorders other than AD.

\section{Acknowledgments}

The authors thank Carina Borén at the Department of Neuropsychiatry, Skaraborg Hospital, Falköping, and Eva Bringman and Dzemila Secic at the Department of Psychiatry and Neurochemistry, Sahlgrenska University Hospital, Mölndal, for excellent technical assistance.

This work was supported by grants from the Swedish Research Council (523-2007-7111, 521-2012-2288, and 2013-2546), the European Research Council (681712), the ALF/LUA 
research grant in Gothenburg (ALFGBG-438631, ALFGBG-139671, and ALFGBG-441051), the Lundberg Foundation, the Torsten and Ragnar Söderberg Foundation, the Lundbeck Foundation, Sahlgrenska University Hospital, Sahlgrenska Academy, Stiftelsen Psykiatriska Forskningsfonden, Stiftelsen Gamla Tjänarinnor, Uppsala Universitets Medicinska Fakultet stiftelse för psykiatrisk och neurologisk forskning, the Alzheimer Foundation, Sweden, the Torsten Söderberg Foundation at the Royal Swedish Academy of Sciences, the Brain Foundation, Sweden, the Knut and Alice Wallenberg Foundation, Frimurarestiftelsen, and the Dementia Association, Sweden.

\section{Disclosure Statement}

K.B. has served as a consultant for Eli Lilly and Roche Diagnostics and at Advisory Boards for Amgen and IBL International. K.B. and H.Z. are co-founders of Brain Biomarker Solutions in Gothenburg AB, a GU venture-based platform company at the University of Gothenburg. The other authors have nothing to disclose.

\section{References}

1 Thrower JS, Hoffman L, Rechsteiner M, Pickart CM: Recognition of the polyubiquitin proteolytic signal. EMBO J 2000;19:94-102.

-2 Haglund K, Sigismund S, Polo S, Szymkiewicz I, Di Fiore PP, Dikic I: Multiple monoubiquitination of RTKs is sufficient for their endocytosis and degradation. Nat Cell Biol 2003;5:461-466.

3 Blennow K, de Leon MJ, Zetterberg H: Alzheimer's disease. Lancet 2006;368:387-403.

4 Perry G, Friedman R, Shaw G, Chau V: Ubiquitin is detected in neurofibrillary tangles and senile plaque neurites of Alzheimer disease brains. Proc Natl Acad Sci USA 1987;84:3033-3036.

5 Mori H, Kondo J, Ihara Y: Ubiquitin is a component of paired helical filaments in Alzheimer's disease. Science 1987;235:1641-1644.

6 Morishima-Kawashima M, Hasegawa M, Takio K, Suzuki M, Titani K, Ihara Y: Ubiquitin is conjugated with amino-terminally processed tau in paired helical filaments. Neuron 1993;10:1151-1160.

7 Tabaton M, Cammarata S, Mancardi G, Manetto V, Autilio-Gambetti L, Perry G, et al: Ultrastructural localization of $\beta$-amyloid, tau, and ubiquitin epitopes in extracellular neurofibrillary tangles. Proc Natl Acad Sci USA 1991; 88:2098-2102.

8 Doran JF, Jackson P, Kynoch PA, Thompson RJ: Isolation of PGP 9.5, a new human neurone-specific protein detected by high-resolution two-dimensional electrophoresis. J Neurochem 1983;40:1542-1547.

$>9$ Wilson PO, Barber PC, Hamid QA, Power BF, Dhillon AP, Rode J, et al: The immunolocalization of protein gene product 9.5 using rabbit polyclonal and mouse monoclonal antibodies. Br J Exp Pathol 1988;69:91-104.

10 Wilkinson KD, Lee KM, Deshpande S, Duerksen-Hughes P, Boss JM, Pohl J: The neuron-specific protein PGP 9.5 is a ubiquitin carboxyl-terminal hydrolase. Science 1989;246:670-673.

11 Liu Y, Fallon L, Lashuel HA, Liu Z, Lansbury PT Jr: The UCH-L1 gene encodes two opposing enzymatic activities that affect $\alpha$-synuclein degradation and Parkinson's disease susceptibility. Cell 2002;111:209-218.

-12 Osaka H, Wang YL, Takada K, Takizawa S, Setsuie R, Li H, et al: Ubiquitin carboxy-terminal hydrolase L1 binds to and stabilizes monoubiquitin in neuron. Hum Mol Genet 2003;12:1945-1958.

13 Lowe J, McDermott H, Landon M, Mayer RJ, Wilkinson KD: Ubiquitin carboxyl-terminal hydrolase (PGP 9.5) is selectively present in ubiquitinated inclusion bodies characteristic of human neurodegenerative diseases. J Pathol 1990;161:153-160.

14 Xie M, Han Y, Yu Q, Wang X, Wang S, Liao X: UCH-L1 inhibition decreases the microtubule-binding function of tau protein. J Alzheimers Dis 2015;49:353-363.

15 Leroy E, Boyer R, Auburger G, Leube B, Ulm G, Mezey E, et al: The ubiquitin pathway in Parkinson's disease. Nature 1998;395:451-452.

16 Carmine Belin A, Westerlund M, Bergman O, Nissbrandt H, Lind C, Sydow O, et al: S18Y in ubiquitin carboxyterminal hydrolase L1 (UCH-L1) associated with decreased risk of Parkinson's disease in Sweden. Parkinsonism Relat Disord 2007;13:295-298.

17 Maraganore DM, Lesnick TG, Elbaz A, Chartier-Harlin M-C, Gasser T, Krüger R, et al: UCHL1 is a Parkinson's disease susceptibility gene. Ann Neurol 2004;55:512-521.

$>18$ Choi J, Levey AI, Weintraub ST, Rees HD, Gearing M, Chin LS, et al: Oxidative modifications and down-regulation of ubiquitin carboxyl-terminal hydrolase L1 associated with idiopathic Parkinson's and Alzheimer's diseases. J Biol Chem 2004;279:13256-13264. 
19 Sultana R, Boyd-Kimball D, Cai J, Pierce WM, Klein JB, Merchant M, et al: Proteomics analysis of the Alzheimer's disease hippocampal proteome. J Alzheimers Dis 2007;11:153-164.

20 Forero DA, Benitez B, Arboleda G, Yunis JJ, Pardo R, Arboleda H: Analysis of functional polymorphisms in three synaptic plasticity-related genes (BDNF, COMT and UCHL1) in Alzheimer's disease in Colombia. Neurosci Res 2006;55:334-341.

21 Xue S, Jia J: Genetic association between ubiquitin carboxy-terminal hydrolase-L1 gene S18Y polymorphism and sporadic Alzheimer's disease in a Chinese Han population. Brain Res 2006;1087:28-32.

22 Zetterberg M, Sjolander A, von Otter M, Palmer MS, Landgren S, Minthon L, et al: Ubiquitin carboxy-terminal hydrolase L1 (UCHL1) S18Y polymorphism in Alzheimer's disease. Mol Neurodegener 2010;5:11.

-23 Kandimalla RJ, Anand R, Veeramanikandan R, Wani WY, Prabhakar S, Grover VK, et al: CSF ubiquitin as a specific biomarker in Alzheimer's disease. Curr Alzheimer Res 2014;11:340-348.

24 Kandimalla RJ, Prabhakar S, Binukumar BK, Wani WY, Sharma DR, Grover VK, et al: Cerebrospinal fluid profile of amyloid beta42 (Abeta42), hTau and ubiquitin in North Indian Alzheimer's disease patients. Neurosci Lett 2011;487:134-138.

25 Blennow K, Davidsson P, Wallin A, Gottfries CG, Svennerholm L: Ubiquitin in cerebrospinal fluid in Alzheimer's disease and vascular dementia. Int Psychogeriatr 1994;6:13-22; discussion 59-60.

26 Oeckl P, Steinacker P, von Arnim CA, Straub S, Nagl M, Feneberg E, et al: Intact protein analysis of ubiquitin in cerebrospinal fluid by multiple reaction monitoring reveals differences in Alzheimer's disease and frontotemporal lobar degeneration. J Proteome Res 2014;13:4518-4525.

27 Blennow K, Hampel H, Weiner M, Zetterberg H: Cerebrospinal fluid and plasma biomarkers in Alzheimer disease. Nat Rev Neurol 2010;6:131-144.

28 Dubois B, Feldman HH, Jacova C, Hampel H, Molinuevo JL, Blennow K, et al: Advancing research diagnostic criteria for Alzheimer's disease: the IWG-2 criteria. Lancet Neurol 2014;13:614-629.

-29 McKhann GM, Knopman DS, Chertkow H, Hyman BT, Jack CR Jr, Kawas CH, et al: The diagnosis of dementia due to Alzheimer's disease: recommendations from the National Institute on Aging-Alzheimer's Association workgroups on diagnostic guidelines for Alzheimer's disease. Alzheimers Dement 2011;7:263-269.

-30 Spiegel J, Pirraglia E, Osorio RS, Glodzik L, Li Y, Tsui W, et al: Greater specificity for cerebrospinal fluid P-tau 231 over P-tau 181 in the differentiation of healthy controls from Alzheimer's Disease. J Alzheimers Dis 2015;49: 93-100.

-31 Bonifati V, Rizzu P, van Baren MJ, Schaap 0, Breedveld GJ, Krieger E, et al: Mutations in the DJ-1 gene associated with autosomal recessive early-onset parkinsonism. Science 2003;299:256-259.

-32 Taira T, Saito Y, Niki T, Iguchi-Ariga SM, Takahashi K, Ariga H: DJ-1 has a role in antioxidative stress to prevent cell death. EMBO Rep 2004;5:213-218.

33 Hong Z, Shi M, Chung KA, Quinn JF, Peskind ER, Galasko D, et al: DJ-1 and $\alpha$-synuclein in human cerebrospinal fluid as biomarkers of Parkinson's disease. Brain 2010;133:713-726.

-34 Gui YX, Liu H, Zhang LS, Lv W, Hu XY: Altered microRNA profiles in cerebrospinal fluid exosome in Parkinson disease and Alzheimer disease. Oncotarget 2015;6:37043-37053.

-35 Blennow K, Wallin A, Ekman R: Neuron specific enolase in cerebrospinal fluid: a biochemical marker for neuronal degeneration in dementia disorders? J Neural Transm Park Dis Dement Sect 1994;8:183-191.

-36 Cutler NR, Kay AD, Marangos PJ, Burg C: Cerebrospinal fluid neuron-specific enolase is reduced in Alzheimer's disease. Arch Neurol 1986;43:153-154.

37 Palumbo B, Siepi D, Sabalich I, Tranfaglia C, Parnetti L: Cerebrospinal fluid neuron-specific enolase: a further marker of Alzheimer's disease? Funct Neurol 2008;23:93-96.

-38 Schmidt FM, Mergl R, Stach B, Jahn I, Gertz HJ, Schonknecht P: Elevated levels of cerebrospinal fluid neuronspecific enolase (NSE) in Alzheimer's disease. Neurosci Lett 2014;570:81-85.

39 Parnetti L, Palumbo B, Cardinali L, Loreti F, Chionne F, Cecchetti R, et al: Cerebrospinal fluid neuron-specific enolase in Alzheimer's disease and vascular dementia. Neurosci Lett 1995;183:43-45.

40 Nooijen PT, Schoonderwaldt HC, Wevers RA, Hommes OR, Lamers KJ: Neuron-specific enolase, S-100 protein, myelin basic protein and lactate in CSF in dementia. Dement Geriatr Cogn Disord 1997;8:169-173.

-41 Johansson P, Mattsson N, Hansson O, Wallin A, Johansson J-O, Andreasson U, et al: Cerebrospinal fluid biomarkers for Alzheimer's disease: diagnostic performance in a homogeneous mono-center population. J Alzheimers Dis 2011;24:537-546.

42 Hansson O, Zetterberg H, Buchhave P, Londos E, Blennow K, Minthon L: Association between CSF biomarkers and incipient Alzheimer's disease in patients with mild cognitive impairment: a follow-up study. Lancet Neurol 2006;5:228-234.

43 Johansson P, Almqvist EG, Johansson JO, Mattsson N, Hansson O, Wallin A, et al: Reduced cerebrospinal fluid level of thyroxine in patients with Alzheimer's disease. Psychoneuroendocrinology 2013;38:1058-1066.

-44 Johansson P, Almqvist EG, Johansson JO, Mattsson N, Andreasson U, Hansson O, et al: Cerebrospinal fluid (CSF) 25-hydroxyvitamin D concentration and CSF acetylcholinesterase activity are reduced in patients with Alzheimer's disease. PLoS One 2013;8:e81989.

45 Johansson P, Aberg D, Johansson JO, Mattsson N, Hansson O, Ahren B, et al: Serum but not cerebrospinal fluid levels of insulin-like growth factor-I (IGF-I) and IGF-binding protein-3 (IGFBP-3) are increased in Alzheimer's disease. Psychoneuroendocrinology 2013;38:1729-1737.

-46 Aberg D, Johansson P, Isgaard J, Wallin A, Johansson JO, Andreasson U, et al: Increased cerebrospinal fluid level of insulin-like growth factor-II in male patients with Alzheimer's disease. J Alzheimers Dis 2015;48:637-646. 
-47 McKhann G, Drachman D, Folstein M, Katzman R, Price D, Stadlan EM: Clinical diagnosis of Alzheimer's disease: report of the NINCDS-ADRDA Work Group under the auspices of Department of Health and Human Services Task Force on Alzheimer's Disease. Neurology 1984;34:939-944.

-48 Roman GC, Tatemichi TK, Erkinjuntti T, Cummings JL, Masdeu JC, Garcia JH, et al: Vascular dementia: diagnostic criteria for research studies. Report of the NINDS-AIREN International Workshop. Neurology 1993;43: 250-260.

49 Erkinjuntti T, Inzitari D, Pantoni L, Wallin A, Scheltens P, Rockwood K, et al: Research criteria for subcortical vascular dementia in clinical trials. J Neural Transm Suppl 2000;59:23-30.

50 Petersen RC: Mild cognitive impairment as a diagnostic entity. J Intern Med 2004;256:183-194.

51 Folstein MF, Folstein SE, McHugh PR: 'Mini-mental state'. A practical method for grading the cognitive state of patients for the clinician. J Psychiatr Res 1975;12:189-198.

-52 Hall S, Ohrfelt A, Constantinescu R, Andreasson U, Surova Y, Bostrom F, et al: Accuracy of a panel of 5 cerebrospinal fluid biomarkers in the differential diagnosis of patients with dementia and/or parkinsonian disorders. Arch Neurol 2012;69:1445-1452.

53 Song S, Jung YK: Alzheimer's disease meets the ubiquitin-proteasome system. Trends Mol Med 2004;10:565570.

54 Diaz-Arrastia R, Wang KK, Papa L, Sorani MD, Yue JK, Puccio AM, et al: Acute biomarkers of traumatic brain injury: relationship between plasma levels of ubiquitin C-terminal hydrolase-L1 and glial fibrillary acidic protein. J Neurotrauma 2014;31:19-25.

55 Lewis SB, Wolper R, Chi YY, Miralia L, Wang Y, Yang C, et al: Identification and preliminary characterization of ubiquitin C terminal hydrolase 1 (UCHL1) as a biomarker of neuronal loss in aneurysmal subarachnoid hemorrhage. J Neurosci Res 2010;88:1475-1484.

-56 Tapiola T, Alafuzoff I, Herukka SK, Parkkinen L, Hartikainen P, Soininen H, et al: Cerebrospinal fluid $\beta$-amyloid 42 and tau proteins as biomarkers of Alzheimer-type pathologic changes in the brain. Arch Neurol 2009;66: 382-389.

57 Buerger K, Ewers M, Pirttila T, Zinkowski R, Alafuzoff I, Teipel SJ, et al: CSF phosphorylated tau protein correlates with neocortical neurofibrillary pathology in Alzheimer's disease. Brain 2006;129:3035-3041.

58 Hampel H, Buerger K, Zinkowski R, Teipel SJ, Goernitz A, Andreasen N, et al: Measurement of phosphorylated tau epitopes in the differential diagnosis of Alzheimer disease: a comparative cerebrospinal fluid study. Arch Gen Psychiatry 2004;61:95-102.

59 Buerger K, Zinkowski R, Teipel SJ, Tapiola T, Arai H, Blennow K, et al: Differential diagnosis of Alzheimer disease with cerebrospinal fluid levels of tau protein phosphorylated at threonine 231. Arch Neurol 2002;59: 1267-1272.

60 Kohnken R, Buerger K, Zinkowski R, Miller C, Kerkman D, DeBernardis J, et al: Detection of tau phosphorylated at threonine 231 in cerebrospinal fluid of Alzheimer's disease patients. Neurosci Lett 2000;287:187-190.

61 Ramont L, Thoannes H, Volondat A, Chastang F, Millet MC, Maquart FX: Effects of hemolysis and storage condition on neuron-specific enolase (NSE) in cerebrospinal fluid and serum: implications in clinical practice. Clin Chem Lab Med 2005;43:1215-1217. 